\title{
A PROBLEM-SPECIFIC GENETIC ALGORITHM FOR PATH PLANNING OF MOBILE ROBOT IN GREENHOUSE
}

\author{
Xuemei Liu ${ }^{1}$, Jin Yuan ${ }^{2,1}$, Kesheng Wang ${ }^{3}$ \\ 'School of Mechanical \& Electronic Engineering, Shandong Agricultural University, China, \\ Email:lxmywj@126.com; ${ }^{2}$ CIMS \& Robot Center of Shanghai University, Shanghai \\ University, China; ${ }^{3}$ Department of Production and Quality Engineering, Norwegian \\ University of Science and Technology, Norway. Email:kesheng.wang@ntnu.no.
}

Abstract: This paper proposes a novel Genetic Algorithms (GAs) approach for a nearoptimal path planning of a mobile robot in a greenhouse. The chromosome encoding features in inverse proportion between research spaces of GAs and complexity of obstacles. The fitness evaluation is designed for both incomplete and complete paths to guide the evolutional direction. The crossover and mutation operators are trimmed to the path planning problem. Two operators were presented to promote the effectiveness of evolution of problem-specific GAs. The simulation results obtained is satisfactory.

Key words: Genetic Algorithms; mobile robot; path planning.

\section{INTRODUCTION}

Use of robots is especially important when the duties performed are potentially harmful for the safety or the health of the workers, or when more conservative issues are granted by robotics. In order to utilize horticulturalrelated vehicles in a greenhouse as robots, it is necessary to plan the work path in advance. The typical task could be described as follows: for two arbitrary points in an area, finding the drivable path between these points minimizing the energy required.

There exist many approaches within Computational Intelligence to solve the problem of finding collision-free paths in the special working places ${ }^{1-3}$,

Please use the following format when citing this chapter:

Liu, Xuemei, Yuan, Jin, Wang, Kesheng, 2006, in International Federation for Information Processing (IFIP), Volume 207, Knowledge Enterprise: Intelligent Strategies In Product Design, Manufacturing, and Management, eds. K. Wang, Kovacs G., Wozny M., Fang M., (Boston: Springer), pp. 211-216. 
which considered the aspects of energy consumption and the length of the paths. However, what most of approaches have in common is that, much time is spent on computing whether the paths are feasible or not, especially the environment with more complicated obstacles. So an advisable approach of path planning with problem-specific GAs is presented.

\section{PATH PLANNING BASED ON GAS}

The proposed GA features its effective encoding, path evaluation and its problem-specific genetic operators. The planed paths are totally independent of the presence or distribution and shape of obstacles.

\subsection{Problem description}

The mobile robot working environment is represented by a 2-dimensional workspace subdivided into quadratic regions at the horizontal and vertical directions according to an appropriate resolution in representing contours of obstacles. Each region named grid is defined by a pair of integer-numbered coordinates. The obstacles are also represented by grids that covered the objects' vertexes. A potential robot path is formed by certain length grids connecting the start grid $\mathrm{S}$ to the target grid $\mathrm{T}$, where $\mathrm{S}$ and $\mathrm{T}$ are represented by their natural coordinates.
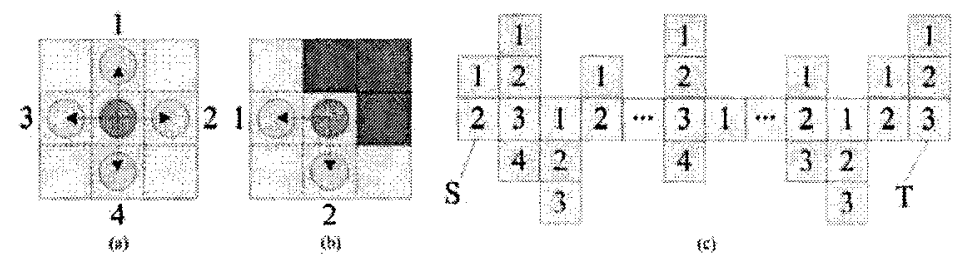

Figure I. (a) The feasible space of one grid with four adjacent regions; (b) One grid with two adjacent regions; (c) The search space under chromosome encoding.

To overcome the huge time expenditure in determining whether a grid zone represents an obstacle or not, the feasible space of a grid was defined generally by 4 adjacent regions, as showed in Fig. $1 \mathrm{a}$. That is, for the current grid (green one), there are four feasible next grids to cover. However, for the grid showed in Fig. 1 b, there are two feasible next grids to cover (the grids in black represents obstacles). Therefore, a potential feasible path could be represented by the variable length chromosome structure, where the sequence contains a number of feasible grids represented by real-valued 
coordinate. Such paths formed by the feasible grids are always collision-free. The length of paths would decrease or increase during the operation of GAs.

The feasible grids contain the research space of GAs, showed in Fig.1c, and correlate with the coverage rate of obstacles. Therefore, the chromosome encoding simplified the research space, and thus speeds up the computation, especially in a complex and congested greenhouse environment.

\subsection{Path initialization and fitness evaluation}

In the GAs, the fitness function guides the evolution direction. A well designed fitness function instructs the right evolution direction and high convergent speed ${ }^{4}$. A robot path was initialized from start grid along feasible space of next grids, so the paths may be either incomplete paths (inaccessible target grid) or complete paths. The evaluation should distinguish incomplete and complete paths and guides the different paths evolving with either category. The GA generates its initial solutions randomly, and evolves solutions starting from the initial population.

The evaluation of path here has two parts. The fitness defined to the incomplete path as $\mathrm{F}_{\mathrm{i}}$, and the complete path is defined as $\mathrm{F}_{\mathrm{c}}$ :

$$
\begin{aligned}
& F_{I}=1000.0 /\left(\left|X_{s}-X_{T}\right|+\left|Y_{s}-Y_{T}\right|\right) \\
& F_{C}=10000.0 \times(1+10.0 / \text { sum }) \\
& \text { sum }=\text { sum }+\left\{\begin{array}{cc}
2 & \text { if } g_{i-1}, g_{i}, g_{i+1} \\
1.5 & \text { else a line }
\end{array}\right.
\end{aligned}
$$

Where, $X_{S}, X_{T}$ are the coordinates of start grid and target grid. The incomplete path closed to target grid has high fitness value in $F_{I}$, and the complete path with shorter sub-path has high fitness value in $\mathrm{F}_{\mathrm{C}}$. In addition, best-1-elitism was implemented to prevent the algorithm from losing the best path and accelerating the convergence of GAs.

\subsection{Operator of Crossover}

The algorithm adopts problem-specific One-Point Crossover. For a chosen two different individuals representing two paths, a feasible intersection points should be collected according to the same grid in two paths. After that, one intersection point is chosen at random, and the subpaths following this point are exchanged (see Fig. 2a). Generally, one path (represented with red) fitness value increases while the other one (represented with black) decreases (see Fig. 2b). 


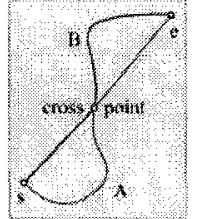

fos

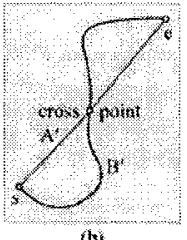

(b)

Figure 2. Operator crossover: (a) before crossover; (b) after crossover.

\subsection{Operator of Mutation}

There are two kinds of mutations due to mutating at different location: the mutation at mid-path and the mutation at end-path. The objects of the former are all paths, which optimize with the direction of shorter path, and the objects of the latter are just incomplete paths, which optimize with the direction of generating the complete paths. However, they are in the same operation mechanism.
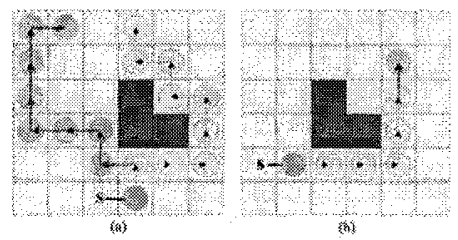

Figure 3. Operator mutation: (a) mutation at mid-path; (b) mutation at end-path.

In Fig. 3a, a grid in complete path was selected randomly. The next grid in its feasible space was selected randomly, and then repeat the process till the length of new path same as the old one. In the Fig. $3 \mathrm{~b}$, the grid was mutated only at the end of path.

\subsection{Operator of Repair}

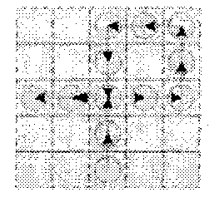

(a)

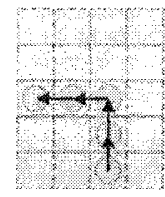

(b)

Figure 4. Operator of repair: (a) before repair; (b) after repair. 
After the operations above have been applied to paths, some enclosed sub-paths may appear (Fig. 4a), which does not contribute to the fitness value of paths, but decrease the mutation and crossover probabilities in the useful sub-paths. The operator of repair can scan the paths to find out the enclosed sub-paths and remove them according to 4 adjacent regions. The result is shown in Fig. 4b.

\subsection{Operator of Tighten}

There are still some undesired sub-paths as shown in Fig. 5b. When GAs reaches its global maximum, the sub-path would be straight, but it would consume much time and it will be difficult to converge with a longer path, making the tighten operation well compromised. First, judging the sub-path meet the condition that there are no obstacles between two arbitrary grids on the path line up in 4 adjacent directions, the sub-path between grids can be tightened. And then using straight grids substitute this sub-path. The subpath does not meet the condition as shown in Fig. 5a. Fig. 5c shows the tighten result.

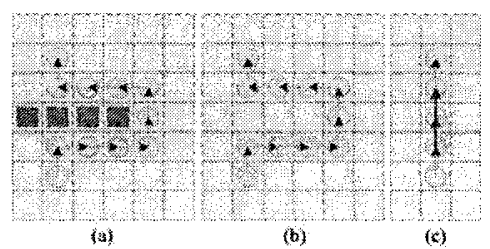

Figure 5. Operator of tighten: (a) path not meet the condition to tighten; (b) path can be tighten; (c) the result of tighten operation.

\section{SIMULATION RESULTS}

Several simulations were conducted about the effectiveness of the proposed GAs. In the simulations, parameters for the proposed GAs were set as follows: population size was 100; probabilities of mutation and crossover were 0.8 and 0.9 ; maximal generations were 100. Fig.6a shows the software interface with tackling the zigzag problem.

The proposed GAs can be easily applied to robot path planning in complicated environment, showed in Fig.6b, which contain 5 arbitrary shapes, and in Fig.6c, which simulating a workplace in a greenhouse. The results of this approach show satisfactory in the meaning of 4 adjacent 
regions. Fig. 6d shows a layout where some potential based path planning approaches and local path planning approaches may be trapped. The result also shows the ability of the proposed GAs to solve maze-like problem.

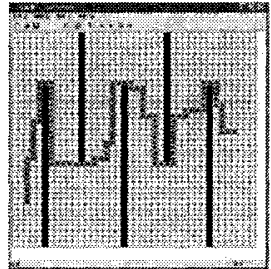

(a)

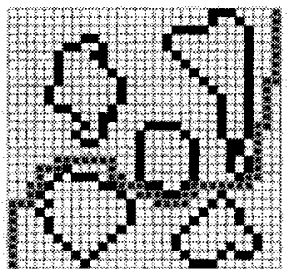

(b)

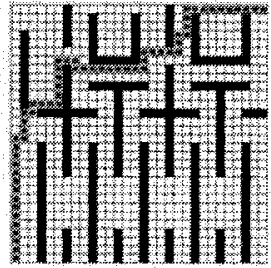

(t)

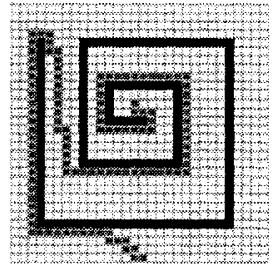

(d)

Figure 6. Simulation examples. (a) A zigzag layout. (b) An arbitrary shape layout. (c) The result in simulating greenhouse environment. (d) The result in maze-like layout.

\section{CONCLUSIONS}

To tackle the movements of mobile robots, a problem-specific GAs for path planning is proposed. Path representation based on feasible space of grids decreases the research space. The fitness evaluation is designed to fit incomplete and complete paths. The crossover and mutation operators are trimmed to the path planning problem. This paper also proposed two problem-specific genetic operators for robot path planning. The effectiveness of the problem-specific GAs is demonstrated by simulation studies. The efficiency in computation time makes the proposed genetic algorithm easily to be applied to real-life applications. The prospective work can be done as the following: (1) To tackle the difficult generation of complete path within the high resolution of environment representation. (2) To avoid the local minima using escaping technique.

\section{REFERENCES}

1. Sugihara, K. et al. (1997). Genetic Algorithms for Adaptive Motion Planning of an autonomous Mobile Robot, Proceedings of the IEEE international Symposium on Computational Intelligence in Robotics and Automation, pp. 138-146.

2. Gemeinder, $M$. et al. (2003), GA-based path planning for mobile robot systems employing an active search algorithm, Applied Soft Computing Journal, (3), p149-158.

3. Gihan, N. et al. (2004), Path planning for a mobile robot using genetic algorithms, Proceedings of ICEEC'04, p 185-189.

4. Wang, K. (2005), Applied Computational Intelligence to Intelligence Manufacturing Systems, Advanced Knowledge International (ISBN 0-9751004-9-1). 